\title{
Reliability of Mesopic Measures of Visual Acuity and Contrast Sensitivity and Their Correlation with Rod and Cone Function in Retinitis Pigmentosa
}

\author{
Ava K. Bittner ${ }^{a, b} \quad$ Mariana C. Ferraz ${ }^{b}$ \\ a Department of Ophthalmology, Stein Eye Institute, University of California, Los Angeles, Los Angeles, CA, USA; \\ ${ }^{\mathrm{b}}$ College of Optometry, Nova Southeastern University, Fort Lauderdale, FL, USA
}

\section{Keywords}

Retinitis pigmentosa $\cdot$ Scotopic $\cdot$ Visual acuity $\cdot$ Contrast sensitivity · Cone sensitivity · Rod function

\begin{abstract}
Background: Mesopic conditions elicit both rod and cone responses, and they are more commonly encountered in daily life than are scotopic conditions; yet visual function outcome measures of mesopic visual acuity (VA) or contrast sensitivity (CS) are rarely evaluated. Objective: In retinitis pigmentosa (RP), we explored whether visual reductions in mesopic versus photopic conditions were correlated with cone or rod function, as well as the between-visit test-retest variability in mesopic measures. Methods: At each of two visits, 22 RP subjects completed mesopic and photopic ETDRS VA and Pelli-Robson chart CS tests obtained with and without a U23 NoIR 4\% transmission filter; testing of perifoveal scotopic cone or rod sensitivity with the AdaptDx; and the Rabin Cone Contrast Test (CCT). Results: A greater CS reduction in mesopic versus photopic conditions was significantly related to absence of scotopic rod function ( $p=$ 0.038 ) or longer self-reported duration of night vision loss $(p=0.044)$. VA reductions $>0.2 \log$ MAR in mesopic versus
\end{abstract}

\section{KARGER}

(๑) 2019 S. Karger AG, Basel

E-Mail karger@karger.com

www.karger.com/ore photopic conditions were significantly related to reduced cone-mediated scotopic sensitivity $(p=0.038)$. Significant predictors of the CCT ratio of S-cone to $\mathrm{M}$ - and L-cone sensitivity were mesopic VA $(p=0.038)$ and absence of AdaptDx rod function ( $p=0.008$ ). Test-retest $95 \%$ coefficients of repeatability were not significantly different when comparing between photopic and mesopic tests of VA $(0.16$ and 0.12 $\log M A R$, respectively) or CS ( 0.21 and $0.24 \log C S$, respectively). Conclusions: Perifoveal scotopic rod and cone function measured with the AdaptDx was significantly correlated with mesopic CS and VA, respectively, which had good, acceptable test-retest repeatability; thus, they appear to be suitable outcome measures to monitor mesopic visual function in clinical practice or trials. RP subjects with reduced mesopic VA and no perifoveal rod function had a greater loss of sensitivity for S-cones than for L-/M-cones.

(c) 2019 S. Karger AG, Basel

\section{Introduction}

Retinitis pigmentosa ( $\mathrm{RP}$ ) primarily affects rod photoreceptor function, but it is also characterized by concurrent or subsequent progressive loss of cone function [1]. 
Some RP patients present with normal rod and cone function in the central retina, while others have loss of both to the same extent, or rod sensitivity loss that precedes cone sensitivity loss throughout the retina in other individuals, while some have only cone function $[2,3]$. These various patterns of rod and cone function loss represent different stages of disease progression for some patients and/or different mechanisms or subtypes of RP, which has been previously documented to be a genetically and phenotypically heterogeneous retinal degenerative disease $[2,3]$. Responses from both rods and cones would be elicited in conditions with mesopic illumination (i.e., dim or low light levels) if both photoreceptors are functional. Mesopic conditions are more often encountered during typical activities of daily living than are scotopic conditions; yet mesopic visual acuity (VA) or contrast sensitivity (CS) tests are rarely included as outcome measures in clinical or research settings. Given the interest in documenting visual changes that may impact the performance of typical daily activities, there is a need to validate testing performed in simulated mesopic illumination as a means of evaluating visual function in dim light conditions among individuals with RP.

Previous research has reported mesopic measures of retinal sensitivity obtained with the microperimeter in $\mathrm{RP}$ [4-7]. However, microperimetry is time-consuming and its test-retest reliability in RP is unknown. A more efficient measure of mesopic central visual function could entail the use of a U23 NoIR 4\% transmission filter while obtaining VA and CS measures, such as with the standard ETDRS and Pelli-Robson letter charts. The use of the U23 NoIR 4\% transmission filter for mesopic VA and CS testing would be low-cost and straightforward to implement across various sites. To validate the use of mesopic measures of VA and CS for the evaluation of individuals with $\mathrm{RP}$, it is important to consider their test-retest reliability. A previous study in which one of the coauthors was involved had reported the $95 \%$ coefficient of repeatability (CR.95) for mesopic VA with the U23 NoIR 4\% transmission filter as very similar to that for photopic VA, i.e., 0.12-0.13 logMAR for $4 \mathrm{RP}$ subjects with a VA $>0.3$ logMAR, but the CR.95 for mesopic VA measured in $12 \mathrm{RP}$ subjects with a VA of 0.3-1.0 logMAR in this study was much higher, i.e., $0.41 \log$ MAR [8]. Similarly, the 95\% coefficients of repeatability determined for mesopic CS with the Pelli-Robson chart were much higher (0.51 $\log C S)$ than those for photopic CS $(0.31 \log C S)$ for RP subjects with a VA of $0.3-1.0 \log$ MAR in that previous study [8]. Given the relatively small number of RP subjects with mild-to-moderate vision loss in that past study and the large test-retest variability for mesopic VA and CS previously documented in RP subjects with moderate vision loss, it remains unclear whether it should be recommended that mesopic VA be included as an additional outcome measure. It is also currently unknown how measures of mesopic VA and CS are related to loss of rod or cone function in RP.

Over 40 years ago, it was initially reported that RP patients had greater loss of sensitivity for blue cones than for green cones [9]. Subsequently, further studies published about 30 years ago found that some patients with the autosomal recessive form of $\mathrm{RP}$, as well as patients with glaucoma or diabetic retinopathy, initially lose shortwavelength cone sensitivity or function (i.e., S-cones) prior to cones that respond to longer wavelengths (i.e., Land M-cones) [10-12]. Around that time, it was hypothesized that this may be related to changes in the optical density of the macular pigment [13], or a mechanism might affect a shared biochemical pathway vulnerability found in both rods and S-cones selectively but not in other cones [10]. However, rod function was not measured in the previous studies, and our recent search of PubMed and similar databases revealed no further research on the possible relationship between loss of S-cone and rod function in RP. S-cones may be more susceptible to changes in the retinal pigment epithelium due to morphologic differences from L- and M-cones. S-cones are smaller, and they have a longer and larger intersegment and deeper innervation projecting further into the subretinal space towards the retinal pigment epithelium [14]. $\mathrm{S}$-cones also have more permeable membranes than Land M-cones [10]. Rods and S-cones are more similar than $\mathrm{L}$ - and $\mathrm{M}$-cones as far as calcium metabolism and proteins, as well as early saturation during psychophysical responses, are concerned [10]. Another potential explanation for the loss of S-cone sensitivity may be the fact that $\mathrm{S}$-cones are the least numerous, representing only $10 \%$ of all cones; therefore, a loss of a few S-cone cells would have a greater impact on visual function. However, previous research has found greater support for metabolic damage as a source of loss of S-cone sensitivity than for postreceptoral causes within the visual system [15].

The aims of the current study were to examine the relationships between scotopic visual function and: (1) VA or CS in mesopic versus photopic conditions in RP, and (2) S-cone sensitivity among individuals with presumed autosomal recessive RP. We anticipated that mesopic measures of VA and CS would be correlated with both photopic and scotopic visual status. Based on previous work in this field, we hypothesized that S-cone sensitivity 
would be correlated with rod function in those with presumed autosomal recessive RP. Furthermore, we were interested in determining and comparing the between-visit test-retest reliability for mesopic and photopic measures of VA and CS. Thus, another motivation for this study was to help validate the use of a protocol for mesopic VA and CS testing in RP.

\section{Subjects and Methods}

\section{Study Design}

Data collection occurred from September 2014 to April 2015. All vision tests were administered or supervised by a single examiner (A.K.B.), and all study outcome measures were repeated at 2 visits (except for 1 participant, who was evaluated twice across 3 visits). The subjects were scheduled to return for the second visit within a 1 - to 2 -week period (mean inter-visit time $=12.7$ days, range 1-42). Each visit lasted approximately 5-6 h. The subjects were offered a lunch voucher to take a break about 2-4 h after the start of the visit, and the tests were made in the same order at each visit. The same examination room and equipment were used for all participants at each visit to ensure that all visual function test conditions were consistent.

\section{Visual Function Tests}

Best corrected VA was measured in each eye using either the refraction findings in a trial frame or habitual spectacle correction if no significant change in refraction was obtained. The Early Treatment of Diabetic Retinopathy Study (ETDRS; Lighthouse International, New York, NY, USA) transilluminated three-chart series were used with a 3-m test distance, which was modified to $1 \mathrm{~m}$ for severely reduced acuities if fewer than 10 letters were originally identified. CS was measured with the Pelli-Robson chart (Metropia, Ltd., UK) at $1 \mathrm{~m}$. Following typical (i.e., photopic, VA and CS) testing, a U23 NoIR $4 \%$ transmission filter was used to simulate mesopic test conditions while measuring binocular VA and CS in the eye with better VA. For both photopic and mesopic VA testing, the room lights were off in a windowless laboratory, with the transilluminated ETDRS chart light turned on, as per standard protocol procedures for VA testing with the ETDRS chart [16]. For photopic CS testing, the room lights were turned on in a windowless laboratory, while the room lights were off for mesopic CS testing, in order to create a further reduction in luminance level to ensure that it was in the mesopic range when confirmed with a light meter positioned behind the NoIR U23 filter directed toward the test chart. During both mesopic and photopic CS testing, a flood light was directed toward the Pelli-Robson chart and away from the subject, with the light between the subject and the chart. An adaptation period of about 2-3 min was implemented consistently across subjects prior to obtaining the mesopic VA and CS measurements.

We determined whether scotopic sensitivity was mediated by cones only (sensitivity $0-3 \log$ units), or by both cones and rods (measurable rod intercept at $3 \log$ units), using the AdaptDx test (MacuLogix, Hummelstown, PA, USA). The rod intercept is defined as the amount of time required for sensitivity recovery to reach a criterion sensitivity level of $5 \times 10^{-3}$ scotopic $\mathrm{cd} / \mathrm{m}^{2}$, which occurs at $3 \log$ units below the brightest stimulus that is presented by the AdaptDx. We administered this test with a $76 \%$ initial flash for bleaching and evaluated the most sensitive perifoveal retinal area located eccentrically at $5^{\circ}$ from central fixation either superiorly, inferiorly, nasally, or temporally, as was determined by results obtained with Humphrey 10-2 static automated perimetry at the same visit prior to the AdaptDx test. The AdaptDx test was stopped after 5-6 min if there was no evidence of rod-mediated dark adaptation (i.e., consistent responses in the sensitivity range of $0-3 \mathrm{log}$ units indicating cone-mediated sensitivity only). Individuals with sensitivities within $3 \log$ units of the brightest stimulus that is presented initially in the AdaptDx test were classified as having a lack of rod response or cone-mediated sensitivity in the current study. We calculated the mean sensitivity across all of their responses during the AdaptDx testing.

The Rabin Cone Contrast Test (CCT; Innova Systems, Burr Ridge, IL, USA) was used to determine the RP patients' sensitivity for each of the three cone types (i.e., blue stimuli for S-cones, green stimuli for M-cones, and red stimuli for L-cones) [17]. The test involves a rapid staircase test that displays 5-16 letters per cone type. The test takes about 5-7 min per cone type and the test distance was about $40-50 \mathrm{~cm}$ with the participants' appropriate near vision correction. At each visit, the CCT was administered twice and all other vision tests were completed once. Each eye was tested individually for photopic (typical) VA, the CCT, and the AdaptDx test, while the Pelli-Robson chart for CS was only completed for the better eye, and photopic and mesopic VA were tested binocularly.

\section{Participants}

A total of 22 participants with a previously confirmed diagnosis of RP were included in this study. Adults over the age of 18 years with RP were eligible for the study if they had better than 1.3 logMAR (i.e., 20/400 or 6/120) best corrected distance VA (photopic) in at least one eye and greater than $20 \%$ loss of Goldmann kinetic visual field area with the III4e test target in at least one eye. Exclusion criteria were (1) vision loss due to ocular diseases other than RP, (2) previous electrostimulation therapy for RP, (3) being nonEnglish speaking, and (4) having a history of excessive bleeding, an implanted cardiac pacemaker, pregnancy, or steroidal systemic medication. No participants had changes in their prescription medications or over-the-counter supplements during the study. The participants' ages ranged from 28 to 60 years (mean 46, SD 10.97) and 12 participants were male. The mean best corrected photopic and mesopic VAs were 0.33 and $0.59 \operatorname{logMAR}$, respectively, across subjects. Nineteen of the 22 participants had measurable Pelli-Robson CS with the 4\% transmission filter, and only 14 of them had measurable cone function with the CCT, since we found that subjects with VA worse than $0.5 \log$ MAR were unable to detect the CCT stimuli. The mean photopic and mesopic CS values were 1.23 and $0.94 \log C S$, respectively, across subjects.

\section{Data Analyses}

For analysis of the CCT results, we used the best score from either visit (i.e., out of 4 tests) for each eye, in order to minimize the test-retest variability noted in the results. For the analysis of the other vision test data for VA, CS, and AdaptDx scotopic cone sensitivity, we used the mean of the results obtained at the 2 visits, for each eye. To compare binocular mesopic VA to monocular cone sensitivity with the CCT or AdaptDx test, we used the 

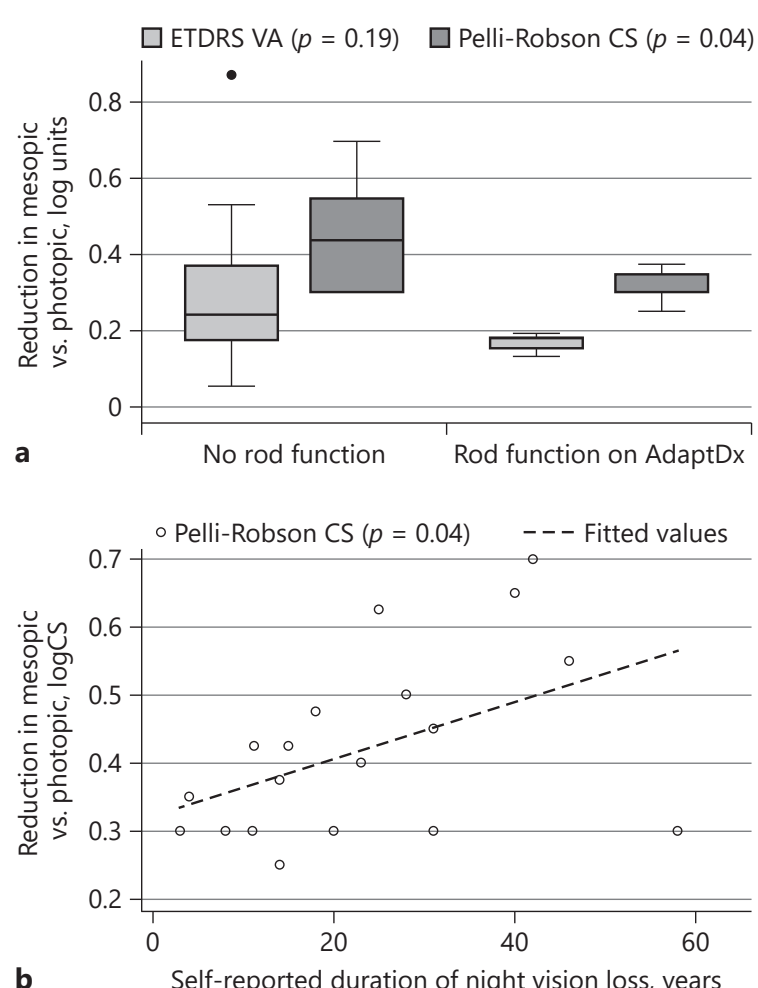

b Self-reported duration of night vision loss, years

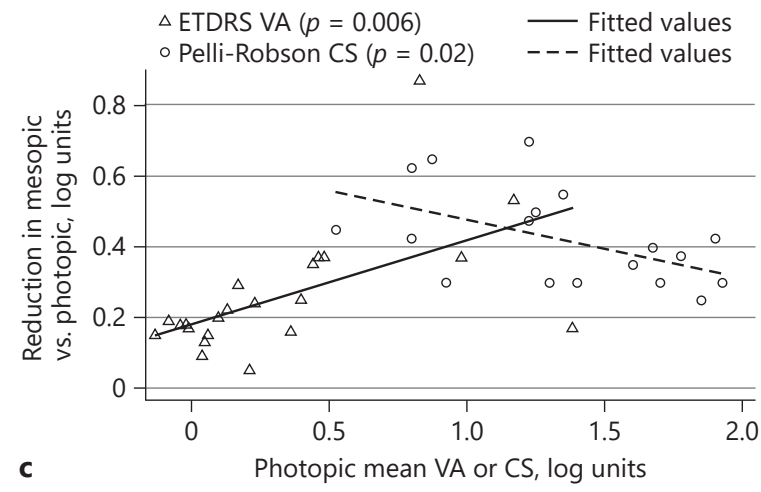

Fig. 1. a Box plot of the reductions in VA and CS in mesopic versus photopic test conditions according to the presence or absence of rod function during AdaptDx testing. The bottom and top of the box are the 25th and 75th percentiles (i.e., the upper and lower quartiles, respectively), and the band near the middle of the box is the 50th percentile (i.e., the median). The ends of the whiskers represent the lowest datum within 1.5 times the interquartile range of the lower quartile, and the highest datum still within 1.5 times the interquartile range of the upper quartile. Any datum not included between the whiskers is plotted as an outlier indicated by a dot. b Scatterplot of the relationship between the reduction in CS in mesopic versus photopic test conditions and the self-reported duration of night vision loss. c Scatterplot of the relationships between the reductions in VA or CS in mesopic versus photopic test conditions versus the mean photopic VA or CS. VA, visual acuity; $\mathrm{CS}$, contrast sensitivity. cone sensitivity values in the eye with better sensitivity for the CCT and AdaptDx test. The data analyses included simple and multivariate linear regressions when each subject contributed data from only one eye, whereas multilevel modeling was used to account for the correlation between subjects' eyes when both were tested, using Stata/IC versions 13.1 and 15.1 (Stata Corp., College Station, TX, USA). The analyses for the CCT that are reported in the results were limited to 12 of the 14 subjects with measurable results who had presumed autosomal recessive RP based on their family history of no affected family members other than siblings (i.e., 2 were excluded since they were presumed to have autosomal dominant RP based on the family history), since our aim was to explore whether they had greater S-cone sensitivity loss than L- or M-cone sensitivity loss, as was previously reported for autosomal recessive RP [10, 12]. Test-retest variability was evaluated with a CR.95 for the tests, with the results in $\log$ units and coefficients of variation $(\mathrm{CoVs})$ for the CCT scores on a $0-100$ scale. Paired $t$ tests were used to compare the between-visit differences for the mesopic versus photopic results for VA or CS to assess whether there was a significant difference in test-retest variability between conditions.

\section{Results}

Greater CS reduction in mesopic versus photopic conditions was significantly related to absence of scotopic rod function $(p=0.038)$, as shown in Figure 1a, or to selfreported greater duration of night vision loss $(p=0.044)$, as shown in Figure 1b. Greater VA reduction in mesopic versus photopic conditions was not significantly related to absence of scotopic rod function $(p=0.19)$, as shown in Figure 1a, or to self-reported greater duration of night vision loss $(p=0.40)$. Figure $1 \mathrm{c}$ shows that there was a significantly greater loss of visual function in mesopic conditions compared to photopic test conditions among subjects with greater photopic visual loss for both VA $(p=0.006)$ and CS $(p=0.02)$. There was a significant relationship between the loss of mean cone-mediated scotopic sensitivity measured with the AdaptDx test and reduced photopic or mesopic VA or CS, as shown in Figure $2 a$ and $b$. VA reductions of $>0.2 \log$ MAR in mesopic versus photopic conditions in $50 \%$ of the subjects were significantly related to reduced cone-mediated scotopic sensitivity by $0.64 \log$ units on average as measured with the AdaptDx test (95\% CI: 0.04, 1.25; $p=0.038$ ), as depicted in Figure 2c. CS reductions of $>0.3 \log C S$ in mesopic versus photopic conditions in $63 \%$ of the subjects were not significantly related to reduced cone-mediated scotopic visual function as measured with the AdaptDx test $(p=$ $0.54)$.

The test-retest CR.95 were not significantly different when comparing between photopic and mesopic tests of 
VA (CR.95: 0.16 and 0.12 logMAR; $p=0.43$ ) or CS (CR.95: 0.21 and $0.24 \log C S ; p=0.68)$, respectively. The test-retest CR.95 was $0.5 \log$ units for the mean sensitivity across subjects with cone-only AdaptDx test responses (i.e., no measurable rod intercept). Bland-Altman graph analyses, shown in Figure 3, revealed that there were no tendencies across subjects to perform better at either the first or the second visit with regard to the cone-mediated AdaptDx responses, or the photopic or mesopic measures of VA or $\mathrm{CS}$, as the mean difference was no more than a letter, and the between-visit variability did not tend to increase with greater visual loss. According to the instructions provided by the test manufacturer for the CCT, reductions in CCT scores are significant when there is a difference of $\geq 15$ points. The within-visit test-retest variability exceeded 15 points for 11 of the measures of cone sensitivity in 8 eyes for 6 of the 14 subjects with measurable CCT results in our study. The between-visit test-retest variability exceeded 15 points for 3 measures of mean cone sensitivity in 2 eyes of 2 subjects in our study. The mean within-visit CoVs for the CCT scores were $11.9 \%$ for L-cones, $12.7 \%$ for M-cones, and 34.8\% for S-cones. The mean betweenvisit $\mathrm{CoV}$ s for the mean CCT scores were $26.0 \%$ for Lcones, $12.8 \%$ for $\mathrm{M}$-cones, and $31.4 \%$ for S-cones. The mean between-visit CoVs for the best CCT scores at each visit were $10.0 \%$ for L-cones, $8.3 \%$ for $\mathrm{M}$-cones, and $14.4 \%$ for S-cones.

Figure 4a shows the significant relationships between photopic VA and the CCT scores for L-cones $(p=0.001)$, M-cones $(p=0.029)$, and S-cones $(p=0.014)$; note the similar slopes and $y$-intercepts for the regression lines for the L- and $\mathrm{M}$-cones, while the regression line for the $\mathrm{S}$-cones indicates greater sensitivity loss across subjects. Mesopic VA, photopic CS, and mesopic CS were also highly significantly related to CCT scores for each of the three cone types (all $p \leq 0.006$ ). In a subgroup analysis of the 12 presumed autosomal recessive RP patients with measurable CCT results, all of the subjects who did not have measurable rod function (i.e., reduced scotopic sensitivity mediated by cones only; $n=8$ ) had greater loss in S-cone sensitivity (for short wavelengths) when comparing the three cone types, while those with remaining rod function $(n=4)$ had either normal S-cone function or relatively equal reductions across all three cone types, as shown in Figure $4 \mathrm{~b}$. One of these subjects with measurable rod function in both eyes had a slower rod response rate (i.e., slightly delayed dark adaptation) and reduced CCT scores for all three cone types in one eye, whereas the better eye had a normal dark adaptation rate and normal CCT scores for all three cone types. In

Relationships between Mesopic Vision and Rod and Cone Function in RP
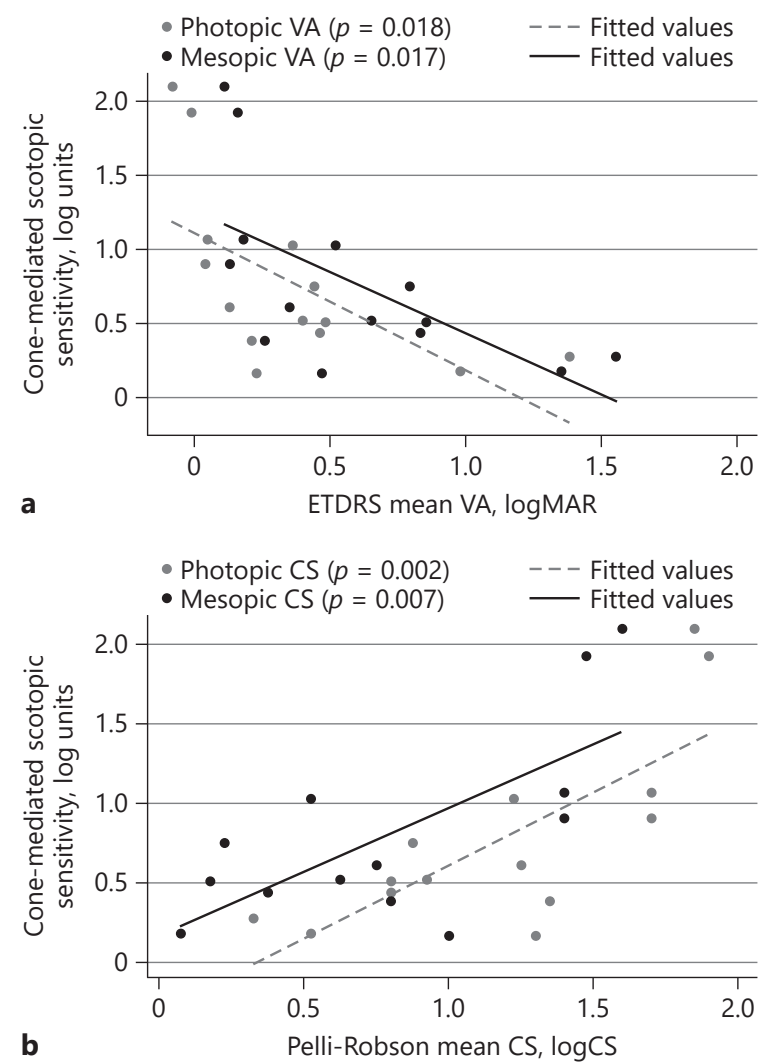

b

Pelli-Robson mean CS, $\log C S$

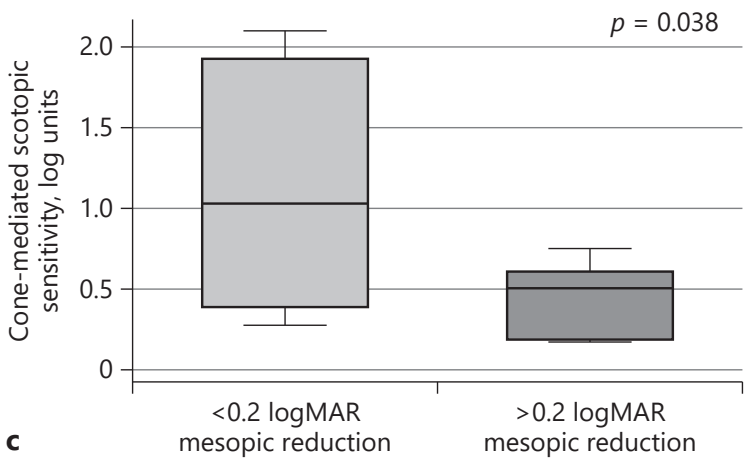

Fig. 2. a, b Scatterplots of the relationships between cone-mediated scotopic sensitivities measured by the AdaptDx test versus the mean photopic or mesopic VA (a) and the mean photopic or mesopic CS (b). c Box plot of the cone-mediated scotopic sensitivities according to whether there was a $<0.2$ or $>0.2 \log$ MAR reduction in $\mathrm{VA}$ in mesopic versus photopic test conditions. VA, visual acuity; CS, contrast sensitivity.

multivariate regression analysis, statistically significant predictors of the ratio of S-cone sensitivity to the mean $\mathrm{M}$ - and L-cone sensitivity were mesopic VA $(p=0.038)$ and absence of scotopic rod function as measured with 


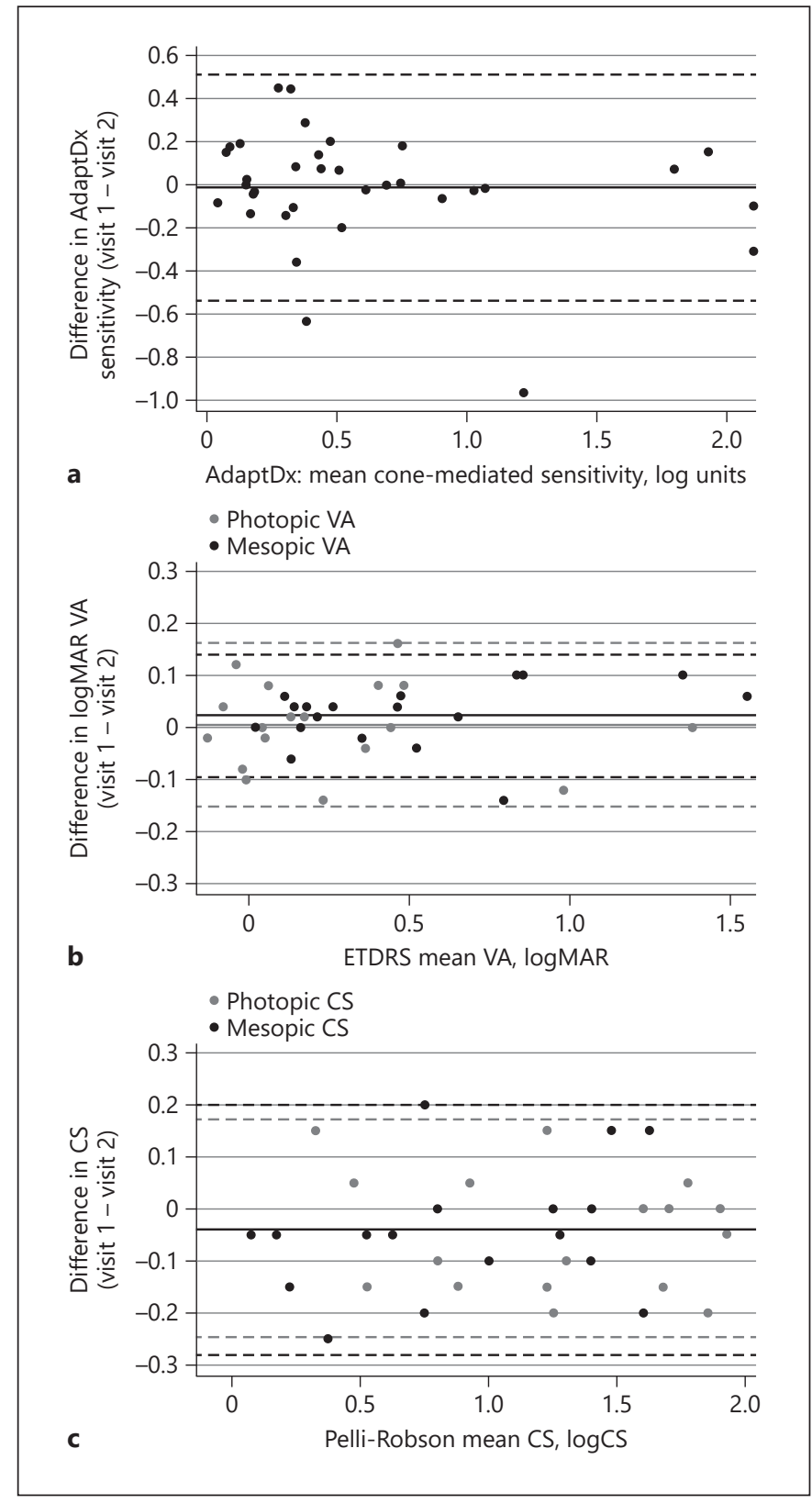

Fig. 3. Bland-Altman-type graphs of the differences in test results (visit 1 - visit 2) versus the mean test results for AdaptDx conemediated sensitivity (a), ETDRS VA (b), and Pelli-Robson CS (c). VA, visual acuity; CS, contrast sensitivity.

the AdaptDx test $(p=0.008)$, which together explained $77.5 \%$ of the variance. When accounting for the absence of AdaptDx test rod function, the measures of photopic VA, photopic CS, or mesopic CS were not significant predictors of the ratio of S-cone to $\mathrm{M}$ - and L-cone sensitivity (each $p \geq 0.09$ ).

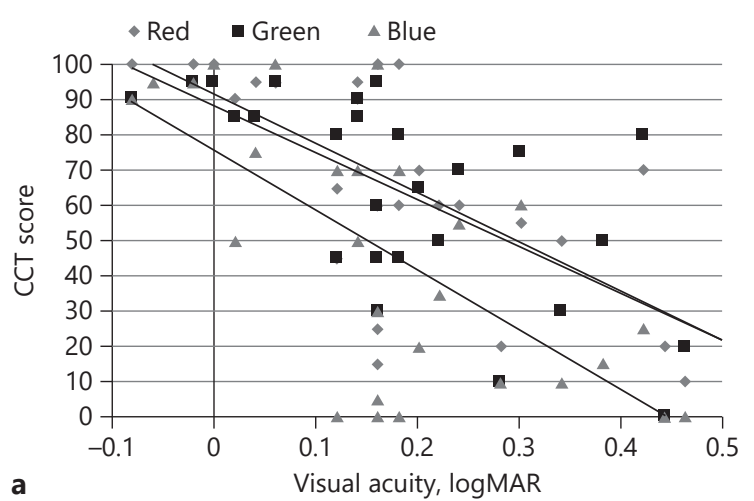

a

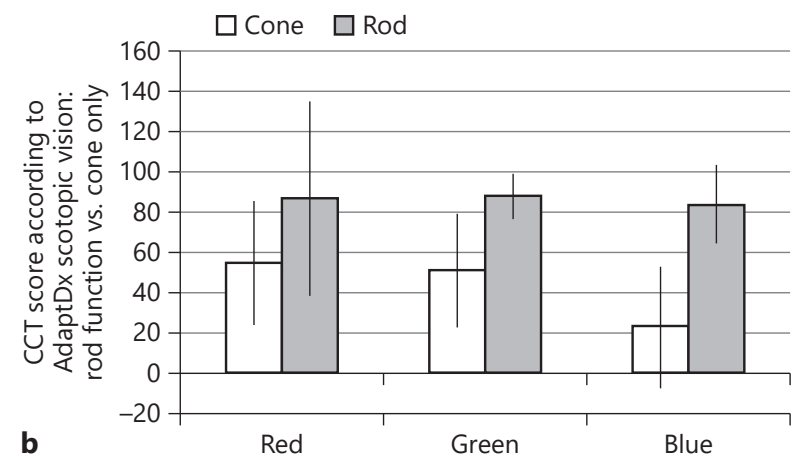

Fig. 4. a Scatterplot of the relationship between the CCT results for each cone type and visual acuity. $\mathbf{b}$ Bar graph of the mean CCT scores for L-, M-, and S-cone sensitivity according to whether scotopic sensitivity was rod- or cone-mediated with the AdaptDx test. The error bars represent SDs. CCT, Cone Contrast Test.

\section{Discussion and Conclusions}

This study revealed that perifoveal scotopic cone function measured with the AdaptDx test in RP was significantly correlated with photopic and mesopic visual function measured with standard VA and CS tests. The RP subjects who had a longer duration of night vision loss or lack of rod-mediated scotopic sensitivity tended to have greater reductions in CS in mesopic versus photopic conditions. The current study also found support for the hypothesis that greater loss of S-cone sensitivity than of Lor M-cone sensitivity is associated with the loss of scotopic rod function and mesopic VA. The AdaptDx test may be helpful to characterize RP patients who have rod- versus cone-mediated scotopic sensitivity at perifoveal locations and to monitor for longitudinal changes outside of the typical test-retest variability of $0.5 \log$ units for RP documented in the present study. The U23 NoIR 4\% 
transmission filter for mesopic VA and CS testing is inexpensive, quick to administer, and easy to implement; therefore, this approach could be readily adopted by several sites in a multicenter trial or across studies as a reliable outcome measure of central mesopic visual function.

A previous study documented much greater betweenvisit test-retest variability than the current study for mesopic measures of VA and CS by about $0.3 \log$ units [10]. One potential factor that may have influenced the lower variability in the current study was the use of a single test examiner for all measurements, whereas the previous study involved several individuals who assisted with the vision testing at different times and may have utilized slightly different approaches during testing (e.g., the amount of time allotted to subjects to adapt to the mesopic filter and provide responses). A key to obtaining reliable test results across visits is the implementation of a strict protocol and consistent methodology during all measurements. This also includes replacement of bulbs in the ETDRS chart or overhead lighting for CS testing as needed during longitudinal studies to maintain consistent chart illumination over time.

There is no ideal or preferred test of scotopic sensitivity or rod function to assess RP patients, as each current commercially available test has its limitations. We did not include the full-field scotopic threshold test in the current study, but it has good test-retest reliability and can be used across a wide range of visual loss, although it is not possible to know which part of the retina is responding with the most sensitivity or whether the most sensitive location changes over time [18]. The AdaptDx test is limited to the test locations available to the instrument (i.e., superior, inferior, nasal, or temporal at 5 or $12^{\circ}$ eccentricity) and requires steady central fixation. We did not use a Dark-Adapted Chromatic (DAC) perimeter (Medmont International Pty Ltd, Nunawading, VIC, Australia) in the present study, which also requires steady central fixation but has a much larger number of test stimulus locations (i.e., up to 164 ) over a 72 by $144^{\circ}$ diameter field for the determination of rod thresholds throughout the retina, with between-visit test-retest reliability in RP that is similar to that of other static automated perimeters and tends to be relatively high (i.e., 9-10 dB) $[19,20]$. Electroretinograms (ERGs) are largely extinguished (i.e., unmeasurable) in most RP patients, including the sample currently reported on here, in which case ERGs were unable to provide information about longitudinal changes in our entire cohort, and rod function may be quantifiable with other tests in the absence of a measurable ERG response [19]. The CCT does not measure scotopic sensitiv-

Relationships between Mesopic Vision and Rod and Cone Function in RP ity, but only RP patients with VA better than $0.5 \log$ MAR had measurable results, and the high test-retest variability of the CCT in the present study indicates that it may not be able to detect typical small, gradual changes in cone sensitivity over time in RP, especially for S-cones, which were most variable. A strength of the current study is the inclusion of people with RP who had a wide range of visual loss, but a limitation is the relatively small sample size; therefore, future clinical trials involving a larger group of participants with RP would be helpful to confirm the current study's findings.

The greater loss of sensitivity for S-cones than L- and $\mathrm{M}$-cones in the absence of functional rods may be likely explained by a combination of multiple mechanisms leading to metabolic damage that have been previously studied in animal models of RP (e.g., structural, toxic, nutritional, trophic, and/or oxidative factors) [21-23]. While it is not currently possible to measure these factors in the retina of human subjects, future clinical trials of therapeutic interventions (e.g., involving neurotrophic or oxidative factors) may be valuable to help explore whether such treatment approaches might impact the function of individual photoreceptor types in people with RP, as well as the possible relationship with these mechanisms. The use of single cell recordings in animal models of RP may be another approach to yield valuable insight into the nature of the loss of specific cone types, thus furthering our knowledge of RP pathophysiology to help direct the development of novel treatment approaches.

\section{Acknowledgements}

The authors wish to thank Samantha Kayser, OD, for her assistance with recruiting and scheduling the study participants, as well as Tracy Topacio, MD, for assistance with data entry from the AdaptDx test.

\section{Statement of Ethics}

The protocol for the study was approved by the Institutional Review Board of the Nova Southeastern University and followed the tenets of the Declaration of Helsinki. Written informed consent was obtained from the subjects after explanation of the nature and possible consequences of the study.

\section{Disclosure Statement}

The authors have no conflicts of interest to declare. The authors alone are responsible for the content and writing of the paper. 


\section{Funding Sources}

This research was funded by National Institutes of Health grant R21 EY023720 awarded to A.K.B.

\section{References}

1 Hartong DT, Berson EL, Dryja TP. Retinitis pigmentosa. Lancet. 2006 Nov;368(9549): 1795-809.

2 Jacobson SG, Roman AJ, Aleman TS, Sumaroka A, Herrera W, Windsor EA, et al. Normal central retinal function and structure preserved in retinitis pigmentosa. Invest Ophthalmol Vis Sci. 2010 Feb;51(2):1079-85.

3 Massof RW, Finkelstein D. Rod sensitivity relative to cone sensitivity in retinitis pigmentosa. Invest Ophthalmol Vis Sci. 1979 Mar; 18(3):263-72.

4 Iftikhar M, Usmani B, Sanyal A, Kherani S, Sodhi S, Bagheri S, et al. Progression of retinitis pigmentosa on multimodal imaging: the PREP-1 study. Clin Exp Ophthalmol. 2019 Jul;47(5):605-13.

5 Sayman Muslubas I, Karacorlu M, Arf S, Hocaoglu M, Ersoz MG. Features of the macula and central visual field and fixation pattern in patients with retinitis pigmentosa. Retina. 2018 Feb;38(2):424-31.

6 Cideciyan AV, Swider M, Aleman TS, Feuer WJ, Schwartz SB, Russell RC, et al. Macular function in macular degenerations: repeatability of microperimetry as a potential outcome measure for ABCA4-associated retinopathy trials. Invest Ophthalmol Vis Sci. 2012 Feb;53(2):841-52.

7 Iftikhar M, Kherani S, Kaur R, Lemus M, Nefalar A, Usmani B, et al. Progression of retinitis pigmentosa as measured on microperimetry: the PREP-1 Study. Ophthalmol Retina. 2018 May;2(5):502-7. Apr;28(4):725-30.

\section{Author Contributions}

A.K.B. is responsible for the study conception, design, conduct, interpretation, statistical analysis, manuscript synthesis (critical revisions), final approval of the manuscript, and accountability for all aspects of the work. M.C.F. is responsible for data interpretations/analyses, drafting the manuscript, final approval of the manuscript, and accountability for all aspects of the work.
8 Kiser AK, Mladenovich D, Eshraghi F, Bourdeau D, Dagnelie G. Reliability and consistency of visual acuity and contrast sensitivity measures in advanced eye disease. Optom Vis Sci. 2005 Nov;82(11):946-54.

9 Sandberg MA, Berson EL. Blue and green cone mechanisms in retinitis pigmentosa. Invest Ophthalmol Vis Sci. 1977 Feb;16(2):14957.

10 Swanson WH, Birch DG, Anderson JL. Scone function in patients with retinitis pigmentosa. Invest Ophthalmol Vis Sci. 1993 Oct;34(11):3045-55.

11 Greenstein VC, Hood DC, Ritch R, Steinberger D, Carr RE. S (blue) cone pathway vulnerability in retinitis pigmentosa, diabetes and glaucoma. Invest Ophthalmol Vis Sci. 1989 Aug;30(8):1732-7.

12 Hood DC, Benimoff NI, Greenstein VC. The response range of the blue-cone pathways: a source of vulnerability to disease. Invest Ophthalmol Vis Sci. 1984 Jul;25(7):864-7.

13 Alexander KR, Hutman LP, Fishman GA. Abnormal foveal spectral sensitivity in retinitis pigmentosa. Invest Ophthalmol Vis Sci. 1987

14 Ahnelt PK, Kolb H, Pflug R. Identification of a subtype of cone photoreceptor, likely to be blue sensitive, in the human retina. J Comp Neurol. 1987 Jan;255(1):18-34.

15 Hood DC, Greenstein VC. Blue (S) cone pathway vulnerability: a test of a fragile receptor hypothesis. Appl Opt. 1988 Mar;27(6):10259.
16 Ferris FL 3rd, Kassoff A, Bresnick GH, Bailey I. New visual acuity charts for clinical research. Am J Ophthalmol. 1982 Jul;94(1):91-6.

17 Rabin J, Gooch J, Ivan D. Rapid quantification of color vision: the cone contrast test. Invest Ophthalmol Vis Sci. 2011 Feb;52(2):816-20.

18 Kiser AK, Mladenovich D, Eshraghi F, Bourdeau D, Dagnelie G. Reliability and consistency of dark-adapted psychophysical measures in advanced eye disease. Invest Ophthalmol Vis Sci. 2006 Jan;47(1):444-52.

19 Bennett LD, Klein M, Locke KG, Kiser K, Birch DG. Dark-Adapted Chromatic Perimetry for Measuring Rod Visual Fields in Patients with Retinitis Pigmentosa. Transl Vis Sci Technol. 2017 Aug;6(4):15.

20 Bennett LD, Metz G, Klein M, Locke KG, Khwaja A, Birch DG. Regional Variations and Intra-/Intersession Repeatability for Scotopic Sensitivity in Normal Controls and Patients with Inherited Retinal Degenerations. Invest Ophthalmol Vis Sci. 2019 Mar;60(4):112231.

21 Koenekoop RK. Why do cone photoreceptors die in rod-specific forms of retinal degenerations? Ophthalmic Genet. 2009 Sep;30(3): $152-4$.

22 Ramachandran PS, Song JY, Bennett J. Exploiting metabolic and antioxidant pathways to maintain vision in blinding disease. J Clin Invest. 2015 Apr;125(4):1390-2.

23 Bovolenta P, Cisneros E. Retinitis pigmentosa: cone photoreceptors starving to death. Nat Neurosci. 2009 Jan;12(1):5-6. 